Ciência Florestal, Santa Maria, v. 27, n. 2, p. 557-569, abr.-jun., 2017

ISSN 1980-5098

\title{
BIOMASSA, CRESCIMENTO E RESPOSTAS ECOFISIOLÓGICAS DE PLANTAS JOVENS DE Bertholletia excelsa BONPL. SUBMETIDAS A DIFERENTES NÍVEIS DE IRRADIÂNCIA
}

\author{
BIOMASS, GROWTH AND ECOPHYSIOLOGICAL RESPONSES OF YOUNG PLANTS OF \\ Bertholletia excelsa BONPL. SUBJECTED TO DIFFERENT LEVELS OF IRRADIANCE
}

\author{
Cristiane Santos do Carmo Ribeiro de Souza ${ }^{1}$ Victor Alexandre Hardt Ferreira dos Santos ${ }^{2}$ \\ Marciel José Ferreira ${ }^{3}$ José Francisco de Carvalho Gonçalves ${ }^{4}$
}

\begin{abstract}
RESUMO
A castanheira-da-amazônia (Bertholletia excelsa Bonpl.) é uma espécie exigente de luz, pois em floresta nativa, a espécie depende da formação de clareiras para alcançar o tamanho reprodutivo e em plantios florestais demonstra rápido crescimento inicial quando exposta a altos níveis de irradiância. No entanto, os aspectos ecofisiológicos dessa espécie sob condições contrastantes de irradiância não foram investigados. Nesse estudo, além das características de crescimento, objetivou-se analisar pela primeira vez o grau de plasticidade das respostas ecofisiológicas de Bertholletia excelsa quando submetida a ambientes de irradiância contrastantes. Plantas jovens de Bertholletia excelsa foram cultivadas em três condições de irradiância: baixa $\left(20-300 \mu \mathrm{mol} \mathrm{m} \mathrm{m}^{-2} \mathrm{~s}^{-1}\right)$, moderada $\left(800-1000 \mu \mathrm{mol} \mathrm{m}^{-2} \mathrm{~s}^{-1}\right)$ e alta $\left(1900-2100 \mu \mathrm{mol} \mathrm{m}^{-2}\right.$ $\left.\mathrm{s}^{-1}\right)$. Foram analisadas características de crescimento, trocas gasosas, teores de pigmentos cloroplastídicos e, no fim do experimento, o acúmulo e partição de biomassa. Plantas jovens de Bertholletia excelsa demonstraram maior acúmulo de biomassa, crescimento e fotossíntese quando expostas aos ambientes de moderada e alta irradiância. A condição de baixa irradiância estimulou maior partição de biomassa para a parte aérea em contraposição ao sistema radicular e maior concentração foliar de pigmentos cloroplastídicos. Bertholletia excelsa possui plasticidade fisiológica sob condições contrastantes de irradiância, sendo o maior crescimento e acúmulo de biomassa, em ambientes de moderada e alta irradiância, promovidos pelo melhor desempenho fotossintético, ao passo que o balanço positivo de carbono sob baixa irradiância é assegurado pelo investimento em estruturas de interceptação e captação de energia.
\end{abstract}

Palavras-chave: Castanheira-da-amazônia; trocas gasosas; crescimento inicial; espécie arbórea tropical.

\section{ABSTRACT}

Brazil nut (Bertholletia excelsa Bonpl.) is a light-demanding species, because in natural forest, the species depending of formation of clearings for reach reproduction size and in forest plantation, showing fast initial growth when exposed to high levels of irradiance. However, the ecophysiological traits of this species under contrasting conditions of irradiance were not investigated. In this study, in addition to growth traits, we investigated for first time the degree of plasticity of ecophysiological response of Bertholletia excelsa when subjected to contrasting irradiance environments. Young plants of Bertholletia excelsa were cultivated under three conditions of irradiance: low $\left(20-300 \mu \mathrm{mol} \mathrm{m}^{-2} \mathrm{~s}^{-1}\right)$, moderate $\left(800-1000 \mu \mathrm{mol} \mathrm{m}^{-2} \mathrm{~s}^{-1}\right)$ and high (1900-2100 $\left.\mu \mathrm{mol} \mathrm{m}^{-2} \mathrm{~s}^{-1}\right)$. We analyzed the growth traits, gas exchange, chloroplastid pigment contents

1 Engenheira Florestal, MSc., Instituto Nacional de Pesquisas da Amazônia (INPA), Av. André Araújo, 2936, CEP 69060-001, Manaus (AM), Brasil.souza_santos_cristiane@hotmail.com

2 Engenheiro Florestal, Mestrando do Programa de Pós-Graduação em Ciências de Florestas Tropicais, Instituto Nacional de Pesquisas da Amazônia (INPA), Av. André Araújo, 2936, CEP 69060-001, Manaus (AM), Brasil. vichardt@hotmail.com

3 Engenheiro Florestal, Dr., Professor Adjunto da Universidade Federal do Amazonas (UFAM), Av. General Rodrigo Octávio, 6200, CEP 69077-000, Manaus (AM), Brasil.mjf.ufam@gmail.com

4 Engenheiro Agrônomo, Dr., Pesquisador Titular do Instituto Nacional de Pesquisas da Amazônia (INPA), Av. André Araújo, 2936, CEP 69060-001, Manaus (AM), Brasil. jfc@inpa.gov.br

Recebido para publicação em 15/07/2015 e aceito em 10/11/2015

Ci. Fl., v. 27, n. 2, abr.-jun., 2017 
and, the end of experiment, the accumulation and partitioning of biomass. Young plants of Bertholletia excelsa showed the highest values of biomass, growth and photosynthesis when exposed to environments of moderate and high irradiance. Low irradiance condition stimulated more biomass partitioning for shoot and chloroplastid pigment contents. Bertholletia excelsa showed physiological plasticity under contrasting conditions of irradiance, the largest growth and biomass accumulation in environments of moderate and high irradiance were promoted by better photosynthetic performance, whereas the positive carbon balance under low irradiance is ensured by investment in structures of interception and energy harvesting.

Keywords: Brazil nut; gas exchange; initial growth; tropical tree.

\section{INTRODUÇÃO}

A castanheira-da-amazônia (Bertholletia excelsa Bonpl.) é uma espécie emergente da floresta ombrófila de terra firme na Amazônia (MORI; PRANCE, 1990). Embora apresente longevidade na sucessão ecológica e não seja espécie pioneira típica, Bertholletia excelsa é considerada clímax exigente de luz, uma vez que sua regeneração natural e crescimento inicial na floresta são dependentes de moderados níveis de irradiância, tais como os observados em clareiras, normalmente, cercadas por dosséis com alturas variadas (SWAINE; WHITMORE, 1988; MORI; PRANCE, 1990; MYERS et al., 2000; MOLL-ROCEK et al., 2014).

Ao considerar as características ecológicas e o potencial econômico desta espécie, diversas iniciativas de plantios de Bertholletia excelsa têm sido desenvolvidas na região Amazônica. Essas experiências se apresentam na forma de enriquecimento de capoeiras, clareiras de exploração florestal e áreas de roçado, recuperação de áreas degradadas, sistemas agroflorestais e também plantios homogêneos com fins de produção de madeira e de frutos (KAINER et al., 1998; D'OLIVEIRA, 2000; PEÑA-CLAROS et al., 2002; TONINI et al., 2008; SOUZA et al., 2010; FERREIRA et al., 2012; MOLL-ROCEK et al., 2014; SCOLES et al., 2014; SCHROTH et al., 2015). Nesses diferentes ambientes de crescimento acima mencionados, embora outros recursos primários, tais como, água e nutrientes, influenciem o estabelecimento dessa espécie no campo (FERREIRA et al., 2009; 2012), a disponibilidade de irradiância tem sido apresentada por diversos autores como fator crucial para o pleno desempenho de Bertholletia excelsa, principalmente durante a fase juvenil (KAINER et al., 1998; ZUIDEMA et al., 1999; HAYASHIDA-OLIVER et al., 2001; PEÑA-CLAROS et al., 2002; SCOLES et al., 2014). Portanto, no que concerne ao ambiente de luz ideal para o pleno crescimento da espécie em plantios ou mesmo sob regeneração natural, muitos avanços foram feitos, por exemplo, maior crescimento de Bertholletia excelsa foi observado em áreas abertas, tais como, roçado de mandioca e agricultura itinerante, quando comparado à introdução dessa espécie em áreas de sub-bosque florestal e capoeiras jovens (KAINER et al., 1998; SCOLES et al., 2014). Contudo, verificamse lacunas no entendimento dos processos de captação de luz e os desdobramentos da captura de elétrons nos sistemas coletores de energia e o ganho de carbono com implicações sobre o crescimento das plantas jovens de Bertholletia excelsa.

Tecnicamente, a abertura do dossel em clareiras de exploração florestal tem proporcionado maiores valores de frequência e crescimento de plantas jovens de Bertholletia excelsa em comparação às áreas adjacentes no sub-bosque altamente sombreado (MOLL-ROCEK et al., 2014). Todavia, são evidentes as necessidades de conhecimentos científicos acerca das características funcionais e estruturais foliares associadas aos mecanismos fotossintéticos que possam determinar a plasticidade de Bertholletia excelsa em função das variações do ambiente lumínico.

Em áreas sob floresta como, por exemplo, sistemas silviculturais de enriquecimento de capoeiras, sub-bosque, clareiras naturais e artificiais ou plantios mistos podem ocorrer aberturas do dossel e, consequentemente, alterações na quantidade e qualidade de energia luminosa limitante ao pleno crescimento e desenvolvimento das mudas após o plantio ou das espécies crescendo nos estratos inferiores ao dossel (KAINER et al., 1998; WIENER, 2010; MOLL-ROCEK et al., 2014). Por outro lado, em situações de plantios em pleno sol (e. g. recuperação de áreas degradadas), o excesso de irradiância somado ao deficit hídrico, alta temperatura e baixa disponibilidade de nutrientes no solo, podem comprometer o desempenho das mudas no campo (SANTOS JÚNIOR et al., 2006). Diante desta sobreposição de fatores que podem limitar o desempenho das espécies no campo, o melhor entendimento das respostas ecofisiológicas das espécies 
arbóreas tropicais ao ambiente de crescimento é fundamental tanto do ponto de vista ecológico quanto para a definição de estratégias mais adequadas de manejo das espécies em iniciativas de reflorestamento e condução de plantios (POORTER, 2001; GONÇALVES et al., 2005; 2012; AZEVEDO; MARENCO, 2012)

A morfologia das espécies arbóreas tropicais durante a fase inicial do desenvolvimento é alterada em função do ambiente de luz predominante e as modificações podem ser observadas, entre outras, na partição da biomassa acumulada em folhas, caule e raízes (POORTER, 2001). Como estratégia para maximizar a interceptação da irradiância, espécies arbóreas tendem a aumentar a área foliar específica e distribuem maior quantidade de biomassa para a parte aérea em ambientes sombreados. Por outro lado, maior quantidade de carbono é alocada no sistema radicular em plantas submetidas às condições de irradiância saturante como alternativa para aumentar a eficiência na absorção de água e nutrientes (ZUIDEMA et al., 1999; KRAUSE et al., 2012; POORTER et al., 2012; TRIPATHI; RAGHUBANSHI, 2014).

Aspectos estruturais foliares associados à captação de energia, e características funcionais foliares relacionadas ao desempenho metabólico também apresentam aclimatação intraespecífica de acordo com a disponibilidade de irradiância no ambiente de crescimento das plantas (GONÇALVES et al., 2012; HALLIK et al., 2012). A concentração de pigmentos cloroplastídicos é utilizada como traço foliar associado à estrutura do aparato fotossintético responsável pela captação e transferência da energia luminosa durante a etapa fotoquímica da fotossíntese (KITAJIMA; HOGAN, 2003; NIINEMETS, 2010; HALLIK et al., 2012; ESTEBAN et al., 2014). Dessa forma, maiores concentrações de pigmentos cloroplastídicos, principalmente, clorofilas $a$ e $b$ são observadas em folhas de plantas adaptadas ao ambiente de baixa irradiância (GONÇALVES et al., 2005; LICHTENTHALER et al., 2007; NIINEMETS, 2010; KRAUSE et al., 2012). Em contrapartida, maiores teores foliares de carotenoides podem conferir apropriada dissipação do excesso de energia em ambientes em que a irradiância atinge valores saturantes, potencialmente desfavoráveis à integridade das membranas biológicas (GONÇALVES et al., 2005; LICHTENTHALER et al., 2007; KRAUSE et al., 2012; LICHTENTHALER et al., 2013).

Além da capacidade das espécies em utilizar e dissipar a energia absorvida, a área foliar específica (AFE) também representa uma modificação necessária ao balanço de carbono positivo e desempenho das espécies arbóreas em ambientes contrastantes de luz. Sendo assim, maiores taxas fotossintéticas em unidade de área foliar têm sido observadas em folhas com reduzida AFE em ambientes com alta disponibilidade de irradiância (KENZO et al., 2015). O aumento no número de camadas de células do mesofilo em folhas com menor AFE representa maior quantidade de tecido apto à realização da fotossíntese e, consequentemente, ocorre um aumento da assimilação líquida de CO2 por unidade de área foliar (OGUCHI et al., 2005; AZEVEDO; MARENCO, 2012).

Considerando o exposto, o objetivo do trabalho foi investigar as características de crescimento e ecofisiológicas de plantas jovens de Bertholletia excelsa submetidas às condições controladas de irradiância variáveis. Portanto, os principais questionamentos investigados foram: i) Quais os efeitos da exposição a diferentes condições de irradiância sobre o crescimento e o acúmulo de biomassa de plantas jovens de Bertholletia excelsa?, e ii) Qual a relação entre as características ecofisiológicas de Bertholletia excelsa e os distintos padrões de crescimento esperados nos diferentes ambientes de luz?

\section{MATERIAL E MÉTODOS}

O experimento foi conduzido em casa de vegetação na Universidade Federal do Amazonas (UFAM), Manaus ( $\left.3^{\circ} 6^{\prime} \mathrm{S}, 59^{\circ} 58^{\prime} \mathrm{W}\right)$. Mudas de Bertholletia excelsa de cinco meses de idade, provenientes do viveiro da Empresa Agropecuária Aruanã S. A. (Itacoatiara-AM), foram selecionadas com valores médios de altura, diâmetro a altura do coleto e número de folhas de $49,4 \pm 7,3 \mathrm{~cm} ; 4,7 \pm 0,5 \mathrm{~mm}$ e $11 \pm 3$ folhas, respectivamente. Durante 60 dias, as mudas foram aclimatadas em casa de sombreamento do Instituto Nacional de Pesquisas da Amazônia (INPA-MCTI) e posteriormente transferidas para recipientes de polietileno com dimensões de 16,7 x $23,8 \mathrm{~cm}$ preenchidos com substrato na proporção 2:1 de terra preta e latossolo amarelo acrescido de calcário e adubação com superfosfato simples, cloreto de potássio, sulfato de amônio e micronutrientes (FTE BR 12) nas dosagens de 0,$891 ; 3,170 ; 0,235 ; 0,151$ e $0,185 \mathrm{~g} \mathrm{dm}^{-3}$, respectivamente.

Após a seleção, aclimatação e transferência de recipientes, as mudas foram submetidas aos 
tratamentos de alta irradiância (AI), moderada irradiância (MI) e baixa irradiância (BI) proporcionados pela exposição das plantas a pleno sol, tela sombrite (50\%) e sombra natural sob uma vegetação arbórea densa, respectivamente. A Radiação Fotossinteticamente Ativa (RFA) instantânea, medida a cada 15 segundos, foi obtida com o sensor quantum externo do modelo Li-COR 6400 do IRGA entre 11:00-13:00 horas em dias de céu claro (pouca nebulosidade) para obter os picos de irradiância. Os valores obtidos para BI variaram entre 20 e $300 \mu \mathrm{mol} \mathrm{m}^{-2} \mathrm{~s}^{-1}$, entre 800 e $1000 \mu \mathrm{mol} \mathrm{m}^{-2} \mathrm{~s}^{-1}$ para MI; e entre 1900 e $2100 \mu \mathrm{mol} \mathrm{m}^{-2} \mathrm{~s}^{-1}$ para AI. As mudas foram irrigadas diariamente.

O crescimento inicial foi avaliado através das características: altura, diâmetro do coleto e número de folhas mensuradas no início do período experimental e 180 dias após a exposição das mudas aos tratamentos, instante em que foi observada a estabilização das taxas de crescimento. As taxas de crescimento absoluto (TCA) em altura e diâmetro do coleto e o ganho foliar (GF) foram determinados pela diferença entre as medidas nos dois períodos. Por sua vez, as taxas de crescimento relativo (TCR) em altura, diâmetro do coleto, e o índice de ganho foliar (IGF) foram calculados conforme Bugbee (1996) e Davanso et al. (2002).

$$
\begin{aligned}
& \mathrm{TCR}=(\operatorname{lnx}-\ln y) /\left(\mathrm{t}_{2}-\mathrm{t}_{1}\right) \\
& \mathrm{IGF}=\left(\frac{\mathrm{NFN}}{\mathrm{NFO}}\right) \times\left(\frac{100}{\mathrm{t}_{2}-\mathrm{t}_{1}}\right)
\end{aligned}
$$

Em que: TCR = taxa de crescimento relativo; $\mathrm{x}=$ valor inicial da variável (diâmetro ou altura); $\mathrm{y}=$ valor final da variável (diâmetro ou altura); $\mathrm{t}_{1}=$ tempo inicial; $\mathrm{t}_{2}=$ tempo final; IGF = índice de ganho foliar; $\mathrm{NFN}=$ número de folhas novas; $\mathrm{NFO}=$ número de folhas originais.

Ao final de 210 dias de experimentação, as mudas de cada tratamento foram seccionadas em raiz, caule e folhas, submetidas à secagem em estufa a $60 \pm 3^{\circ} \mathrm{C}$ durante 72 horas e então foram obtidos os valores de massa seca total (MST), foliar (MSF), caulinar (MSC) e radicular (MSR). Em função dos valores de massa seca total foram obtidas as frações de massa foliar (FMF), caulinar (FMC) e radicular (FMR). Adicionalmente, foi calculada a razão entre a massa seca aérea e radicular (MSA:MSR).

A área foliar específica (AFE) foi determinada aos 0; 45 e 205 dias após o início do experimento. Dez discos foliares de área conhecida foram coletados em cinco plantas de cada tratamento, acondicionados em sacos de papel e submetidos à estufa $\left(70^{\circ} \mathrm{C}\right)$ por um período de $72 \mathrm{~h}$ para posterior determinação da relação área foliar fresca $\left(\mathrm{cm}^{2}\right)$ : massa foliar seca (g) (EVANS; POORTER, 2001).

A determinação das taxas de fotossíntese líquida (A), respiração no escuro (Rd), condutância estomática (gs) e transpiração (E) foi realizada aos 0; 20; 50; 70; 85; 100 e 120 dias de submissão aos tratamentos de irradiância, momento no qual os valores de A, Rd, gs e E permaneceram constantes. As medidas foram realizadas por meio do analisador de gás a infravermelho (LI-6400, LI-COR, Lincoln, USA), conforme metodologia descrita por Santos Júnior et al. (2006). Os dados foram coletados entre 8h e $12 \mathrm{~h}$, em folhas completamente expandidas, em bom estado fitossanitário e situadas no terço médio das plantas. As medições foram realizadas aleatoriamente, sendo a obtenção dos dados realizada sempre em diferentes horários para cada tratamento. $\mathrm{O}$ analisador de gás a infravermelho foi ajustado para trabalhar com densidade de fluxo de fótons (PPFD) saturante de $2000 \mu \mathrm{mol} \mathrm{m}^{-2} \mathrm{~s}^{-1}$, concentração de $\mathrm{CO}_{2}$ de $380 \pm 4$ $\mu \mathrm{mol} \mathrm{mol}{ }^{-1}$, temperatura do bloco de $31 \pm 1^{\circ} \mathrm{C}$ e vapor de $\mathrm{H}_{2} \mathrm{O}$ de $21 \pm 1 \mathrm{mmol} \mathrm{mol}^{-1}$.

Os teores foliares de pigmentos cloroplastídicos foram determinados aos 0; 45 e 205 dias de experimentação em folhas completamente expandidas e sadias de acordo com o método descrito por Lichtenthaler e Wellburn (1983) e calculados conforme as equações descritas por Hendry e Price (1993). Foram determinados os teores de clorofila $a(\mathrm{Chl} a)$, clorofila $b(\mathrm{Chl} b)$, e carotenoides $\left(\mathrm{C}_{\mathrm{c}+\mathrm{x}}\right)$ e, partir desses valores, foram calculados, na unidade de massa foliar, os teores de clorofila total $\left(\mathrm{Chl}_{\text {total }}\right)$, a razão entre clorofila $a$ e clorofila $b(\mathrm{Chl} a / b)$ e a razão entre clorofila total e carotenoides $\left(\mathrm{Chl}_{\text {total }} / \mathrm{C}_{\mathrm{c}+\mathrm{x}}\right)$.

A determinação de pigmentos cloroplastídicos e AFE foi realizada aos 0; 45 e 205 dias em função da demanda por elevada quantidade de material vegetal (folhas). Aos 180 dias o material vegetal era insuficiente para as análises de pigmentos e AFE do tratamento BI, assim, as análises foram realizadas somente aos 205 dias. Em consequência, as características associadas ao acúmulo e partição de biomassa, que exigem análise destrutiva, foram observadas aos 210 dias de experimentação. 
O delineamento experimental utilizado foi o inteiramente casualizado com três tratamentos: $\mathrm{BI}$ baixa irradiância (20 e $300 \mu \mathrm{mol} \mathrm{m}^{-2} \mathrm{~s}^{-1}$ ), MI - moderada irradiância (800 e $1000 \mu \mathrm{mol} \mathrm{m}^{-2} \mathrm{~s}^{-1}$ ) e AI - alta irradiância (1900 e $2100 \mu \mathrm{mol} \mathrm{m}^{-2} \mathrm{~s}^{-1}$ ) com 15 repetições, sendo cada repetição representada por uma planta jovem de Bertholletia excelsa. Entre as 15 repetições, cinco plantas foram utilizadas para a obtenção dos valores de AFE e teor de pigmentos cloroplastídicos, enquanto os dados de trocas gasosas (A, Rd, gs e E) foram obtidos nas demais plantas por tratamento. Os resultados de crescimento, acúmulo e partição de biomassa foram previamente submetidos aos testes de normalidade de Lilliefors e homogeneidade de variância de Levene e, em seguida analisados mediante ANOVA One-Way e teste de Tukey $(P<0,05)$. Os valores de AFE, teor de pigmentos cloroplastídicos e trocas gasosas (A, Rd, gs e E) foram submetidos aos testes de normalidade de Lilliefors, homogeneidade de variância de Levene, esfericidade de Mauclhy e analisados mediante ANOVA de medidas repetidas no tempo. Em seguida foi aplicado o teste de Tukey $(P<0,05)$. As análises foram realizadas no software Statistica versão 9.0 para Windows (STATSOFT, 2010).

\section{RESULTADOS E DISCUSSÃo}

Nesse trabalho, acrescentaram-se evidências sobre características de crescimento e acúmulo de biomassa de Bertholletia excelsa em ambientes com disponibilidade de irradiâncias variáveis. Além disso, pela primeira vez, foram obtidos dados a respeito de aspectos estruturais e funcionais foliares de plantas jovens de Bertholletia excelsa submetidas a níveis de baixa, moderada e alta irradiância.

$\mathrm{O}$ acúmulo e partição de biomassa das plantas jovens de Bertholletia excelsa foram influenciados pela exposição aos diferentes níveis de irradiância (Tabela 1). Em condições de baixa irradiância, o acúmulo de biomassa seca total, foliar, caulinar e radicular foi reduzido quando comparado aos demais tratamentos, com destaque para a massa seca radicular (MSR) que foi, aproximadamente, 9 vezes inferior. A partição de biomassa entre folhas, caule e raízes também diferiu na comparação dos tratamentos, uma vez que em plantas submetidas aos tratamentos de elevada e moderada irradiância foram observadas maiores proporções de massa radicular e menor proporção de massa caulinar e foliar que as plantas jovens em baixa irradiância. Como resultado da maior partição de biomassa para folhas e caule, a proporção de biomassa aérea nas plantas do tratamento BI foi aproximadamente quatro vezes superior aos demais tratamentos. De forma semelhante, outros autores relataram menor acúmulo de biomassa e maior proporção de biomassa investida acima do solo em várias espécies florestais, dentre elas Bertholletia excelsa, crescendo em ambientes de baixa disponibilidade de irradiância (ZUIDEMA et al., 1999; HAYASHIDA-OLIVER et al., 2001; GONÇALVES et al., 2012; KRAUSE et al., 2012; TRIPATHI; RAGHUBANSHI, 2014). A maior proporção de biomassa em órgãos da parte aérea tem sido associada a estratégias de aperfeiçoamento da captação de luz em ambientes de baixa irradiância. Por outro lado, o maior investimento de biomassa em raízes aprimora a captação de água e nutrientes que podem ser os fatores de crescimento mais limitantes em condições de alta disponibilidade de energia luminosa (POORTER, 2001; POORTER et al., 2012).

Durante 180 dias de experimentação, os valores de TCA em altura e diâmetro variaram entre 0,76 e 1,35 cm mês ${ }^{-1}$ e 0,18 e $0,76 \mathrm{~mm} \mathrm{mês}^{-1}$, respectivamente (Figura 1). O ambiente de irradiância influenciou significativamente as taxas de crescimento absoluto em altura $(\mathrm{P}<0,01)$ e diâmetro $(\mathrm{P}<0,01)$ de plantas jovens de Bertholletia excelsa (Figura 1). Nesse sentido, foi observado que em plantas crescendo em condições de BI os valores de TCA em altura e diâmetro foram, respectivamente, 2 e 4 vezes inferiores ao demais tratamentos. Considerando os valores de TCR, a amplitude observada foi de 0,014 a $0,027 \mathrm{~cm} \mathrm{~cm}^{-1}$ mês ${ }^{-1}$ para altura e 0,036 a $0,119 \mathrm{~mm} \mathrm{~mm}^{-1}$ mês $^{-1}$ para diâmetro (Figura 1). Novamente, os maiores valores foram observados em plantas crescendo nos ambientes de moderada (MI) e alta irradiância (AI), com TCR em altura $(\mathrm{P}<0,01)$ e diâmetro $(\mathrm{P}<0,01)$, respectivamente, 2 e 3 vezes superiores aos observados no tratamento BI. O ganho foliar foi positivo nas três condições avaliadas, porém, os valores observados em MI e AI foram 3 e 3,5 vezes superiores aos verificados em BI, respectivamente. A amplitude total para o IGF foi de $0 \%$ a $16 \%$ e os valores médios observados em AI e MI foram aproximadamente 3 vezes superiores aos obtidos em BI. Por ser uma espécie clímax exigente de luz, evidências sobre o crescimento de Bertholletia excelsa em diferentes ambientes de luz, tais como, condições controladas em viveiro (ZUIDEMA et al., 1999; HAYASHIDA-OLIVER et al., 2001), regeneração artificial e natural em clareiras (OLIVEIRA, 2000; MOLL-ROCEK et al., 2014), plantios de enriquecimento (capoeiras e roçados) e plantios sob pleno sol 
TABELA 1: Massa seca total (MST), caulinar (MSC), radicular (MSR), foliar (MSF), fração de massa caulinar (FMC), foliar (FMF) e radicular (FMR) e relação entre biomassa aérea e radicular (MSA:MSR) em plantas jovens de Bertholletia excelsa submetidas a ambientes contrastantes de irradiância.

TABLE 1: Total dry matter (MST), stem (MSC), root (MSR), leaf (MSF), fraction of stem (FMC), leaf (FMF) and root (FMR) matter and ratio shoot and root (MSA:MSR) in young plants of Bertholletia excelsa subjected to contrasting environments of irradiance.

\begin{tabular}{cccc}
\hline \multirow{2}{*}{ Biomassa } & \multicolumn{3}{c}{ Tratamento } \\
\cline { 2 - 4 } & Baixa Irradiância & Moderada Irradiância & Alta Irradiância \\
\hline MST $(\mathrm{g})$ & $8,17 \pm 0,78 \mathrm{~b}$ & $28,25 \pm 2,96 \mathrm{a}$ & $28,87 \pm 2,26 \mathrm{a}$ \\
MSC $(\mathrm{g})$ & $4,87 \pm 0,41 \mathrm{~b}$ & $13,57 \pm 1,30 \mathrm{a}$ & $11,91 \pm 0,91 \mathrm{a}$ \\
MSR $(\mathrm{g})$ & $1,48 \pm 0,24 \mathrm{~b}$ & $11,04 \pm 2,05 \mathrm{a}$ & $12,86 \pm 1,27 \mathrm{a}$ \\
MSF $(\mathrm{g})$ & $1,82 \pm 0,25 \mathrm{~b}$ & $3,64 \pm 0,27 \mathrm{a}$ & $4,10 \pm 0,37 \mathrm{a}$ \\
FMC & $0,60 \pm 0,02 \mathrm{a}$ & $0,49 \pm 0,02 \mathrm{~b}$ & $0,42 \pm 0,07 \mathrm{~b}$ \\
FMF & $0,22 \pm 0,01 \mathrm{a}$ & $0,14 \pm 0,01 \mathrm{~b}$ & $0,14 \pm 0,01 \mathrm{~b}$ \\
FMR & $0,18 \pm 0,02 \mathrm{~b}$ & $0,37 \pm 0,03 \mathrm{a}$ & $0,44 \pm 0,02 \mathrm{a}$ \\
MSA:MSR & $5,19 \pm 0,67 \mathrm{a}$ & $1,81 \pm 0,20 \mathrm{~b}$ & $1,31 \pm 0,11 \mathrm{~b}$ \\
\hline
\end{tabular}

Em que: Os valores são médias \pm erro padrão $(n=15)$; médias seguidas pelas mesmas letras não diferem entre si pelo teste de Tukey $(\mathrm{P}<0,05)$. Baixa Irradiância $\left(20-300 \mu \mathrm{mol} \mathrm{m} \mathrm{m}^{-2} \mathrm{~s}^{-1}\right)$, Moderada Irradiância $\left(800-1000 \mu \mathrm{mol} \mathrm{m} \mathrm{m}^{-2} \mathrm{~s}^{-1}\right) \mathrm{e}$ Alta Irradiância $\left(1900-2100 \mu \mathrm{mol} \mathrm{m}^{-2} \mathrm{~s}^{-1}\right)$.

(KAINER et al., 1998; PEÑA-CLAROS et al., 2002; SOUZA et al., 2010; SCOLES et al., 2014), sugerem que o pleno crescimento de Bertholletia excelsa é dependente da disponibilidade de irradiância e corroboram os valores observados no presente estudo. Em áreas de roçado de mandioca, com maior disponibilidade de luz, o incremento médio anual em altura de Bertholletia excelsa após 6 anos foi, aproximadamente, 40 vezes superior ao observado em plantas da mesma espécie no sub-bosque de castanhais com abertura do dossel menor que $10 \%$ (SCOLES et al., 2014).

Os valores médios de AFE foram influenciados pelos tratamentos $(\mathrm{P}<0,05)$ e período experimental $(\mathrm{P}<0,01)$ (Figura 2). Em todos os tratamentos foram observadas reduções da área foliar específica ao final do experimento. Maiores reduções, entre o início e 205 dias de experimentação, foram observadas em plantas submetidas aos tratamentos AI (58\%) e MI (34\%) quando comparados ao BI (12\%). Dessa forma, os valores de AFE em plantas submetidas ao tratamento BI durante 205 dias foram $35 \%$ e $20 \%$ superiores aos observados em AI e MI, respectivamente. Maiores valores de área foliar e menor espessura do mesofilo, com o consequente aumento de AFE, aprimoram a área de interceptação da irradiância em ambientes com baixa disponibilidade do recurso (POORTER et al., 2012; TRIPATHI; RAGHUBANSHI, 2014).

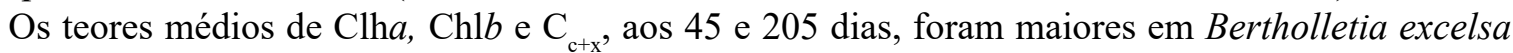
crescendo sob condições de baixa irradiância e, consequentemente, a concentração de $\mathrm{Chl}_{\text {total }}$, aos 205 dias, foi 3,6 e 4,5 vezes superior aos tratamentos MI e AI, respectivamente (Figura 3). Em condições de alta irradiância há um desequilíbrio entre a síntese e degradação dos pigmentos cloroplastídicos, com predominância da degradação por foto-oxidação, tal como observado em MI e AI (GONÇALVES et al., 2005; LICHTENTHALER et al., 2007; KRAUSE et al., 2012). Os níveis de irradiância nos tratamentos AI e MI proporcionaram aumento da razão Chla/b e, aos 205 dias, os valores foram, respectivamente, $56 \%$ e $47 \%$ maiores que os observados antes da exposição aos tratamentos. Todavia, os valores de Chla/b foram menores e permaneceram constantes até o final do experimento no tratamento BI, uma vez que ocorreu o aumento proporcional nas concentrações de Chla e Chl $b$. A clorofila $b$ está presente, principalmente, nos complexos coletores de luz dos fotossistemas I e II (CCLI e CCLII), no entanto a clorofila $a$ está presente nos complexos coletores de luz e nos centros de reação dos fotossistemas. Nesse contexto, um aumento dos complexos coletores de luz, aprimorando a captação da energia disponível, pode estar associado ao baixo 


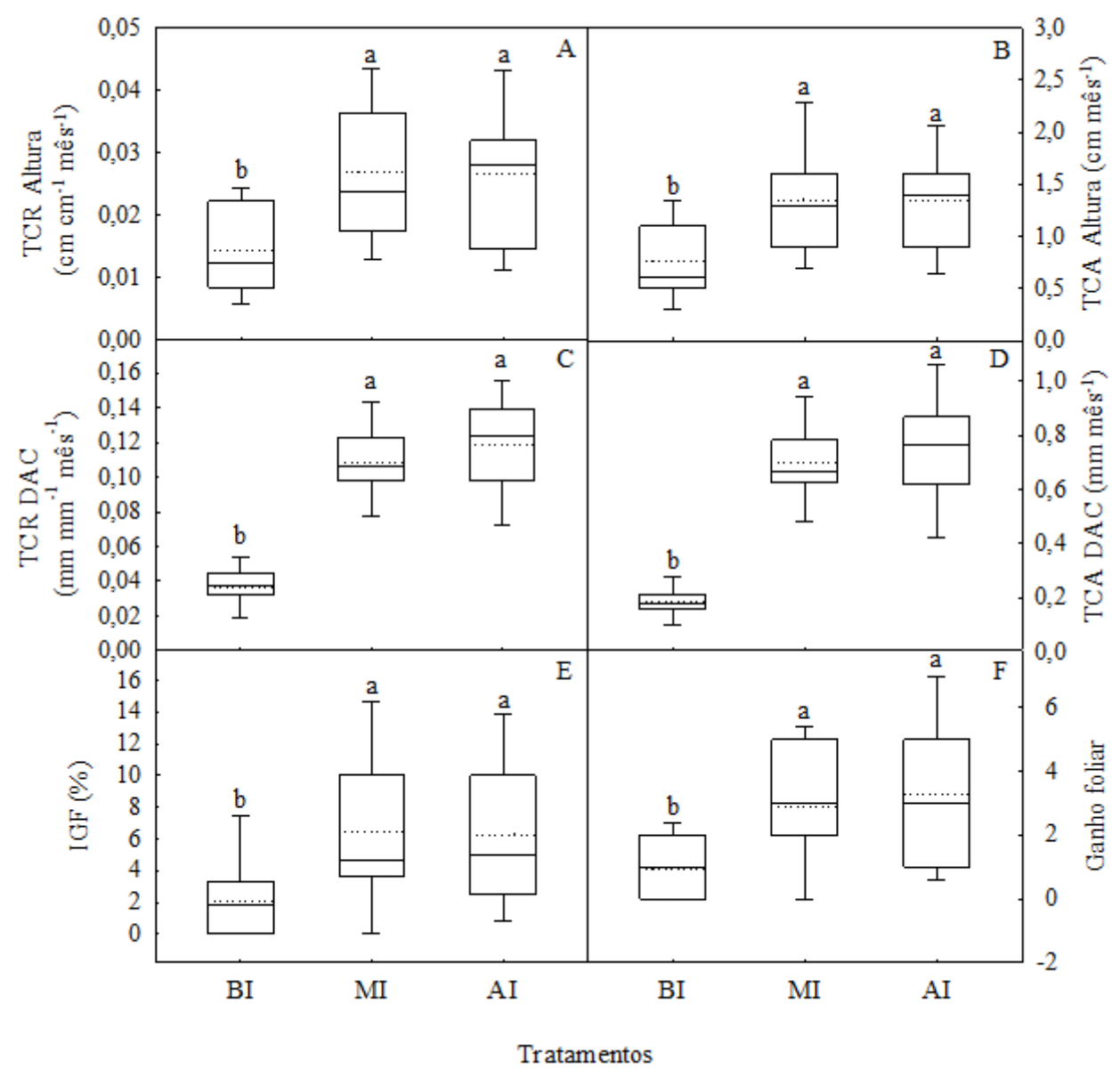

FIGURA 1: Taxas de crescimento relativo (A) e absoluto (B) em altura, taxas de crescimento relativo (C) e absoluto (D) em diâmetro do coleto - DAC, índice de ganho foliar (E) e ganho foliar (F) de plantas jovens de Bertholletia excelsa submetidas a condições contrastantes de irradiância. Linha contínua no interior do box - mediana, linha pontilhada no interior do box - média, área do box-plot $=50 \%$ dos dados, com barras de erro exibindo valores do $5^{\circ}$ e $95^{\circ}$ percentis. $\mathrm{AI}=$ alta irradiância $\left(1900-2100 \mu \mathrm{mol} \mathrm{m}^{-2} \mathrm{~s}^{-1}\right)$; MI = moderada irradiância $\left(800-1000 \mu \mathrm{mol} \mathrm{m} \mathrm{m}^{-2}\right.$ $\left.\mathrm{s}^{-1}\right)$ e $\mathrm{BI}=$ baixa irradiância $\left(20-300 \mu \mathrm{mol} \mathrm{m}^{-2} \mathrm{~s}^{-1}\right)(\mathrm{n}=15)$. Médias seguidas pelas mesmas letras não diferem entre si pelo teste de Tukey $(\mathrm{P}<0,05)$.

FIGURE 1: Absolute (A) and relative (B) growth rates in height, absolute (C) and relative (D) growth rates in diameter at collar height - DAC, leaf gain index $(\mathrm{E})$ and foliar gain $(\mathrm{F})$ of young plants of Bertholletia excelsa subjected to contrasting conditions of irradiance. Continuous line inside the box - median, dashed line inside the box - mean, box-plot area $=50 \%$ of the data, with error bars showing of $5^{\text {th }}$ and $95^{\text {th }}$ percentile values. AI $=$ high irradiance $(1900-2100 \mu \mathrm{mol}$ $\left.\mathrm{m}^{-2} \mathrm{~s}^{-1}\right) ; \mathrm{MI}=$ moderate irradiance $\left(800-1000 \mu \mathrm{mol} \mathrm{m}^{-2} \mathrm{~s}^{-1}\right)$ and $\mathrm{BI}=$ low irradiance $(20-300$ $\left.\mu \mathrm{mol} \mathrm{m} \mathrm{m}^{-2} \mathrm{~s}^{-1}\right)(\mathrm{n}=15)$. Means followed by the same letters do not differ according to Tukey test $(\mathrm{P}<0.05)$.

valor da relação Chla/b no tratamento BI (KITAJIMA; HOGAN, 2003; NIINEMETS, 2010; HALLIK et al., 2012; ESTEBAN et al., 2014). A concentração de $\mathrm{Ch}_{\text {total }}$ relativa aos teores de $\mathrm{C}_{\mathrm{c}+\mathrm{x}}$ reduziu 2 e 1,6 vezes no decorrer do período experimental nos tratamentos AI e MI, respectivamente. De forma semelhante à relação Chla/b, a razão $\mathrm{Chl}_{\text {total }} / \mathrm{C}_{\mathrm{c}+\mathrm{x}}$ permaneceu constante em BI. Baixos valores de $\mathrm{Ch} l_{\text {total }} / \mathrm{C}_{\mathrm{c}+\mathrm{x}} \mathrm{em}$ ambientes de moderada e alta irradiância também foram observados por outros autores (HANSEN et al., 2002; GONÇALVES et al., 2005; HALLIK et al., 2012). A elevada proporção de carotenoides por clorofila está em grande medida associada à maior eficiência na dissipação do excesso de energia luminosa nos 


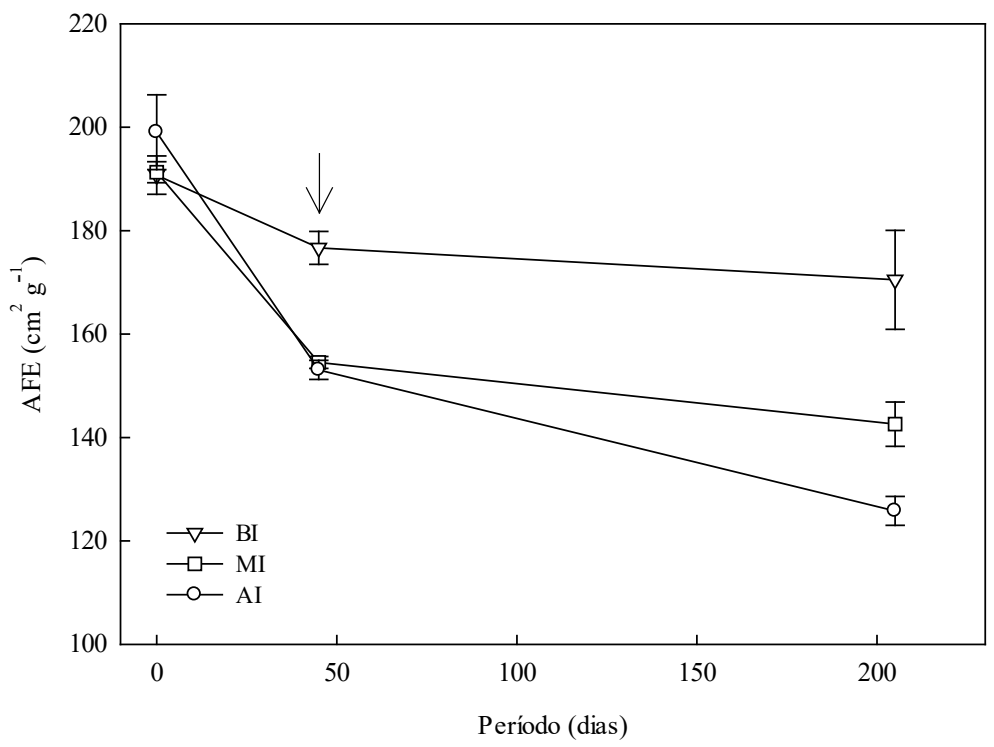

FIGURA 2: Área foliar específica (AFE) em plantas jovens de Bertholletia excelsa submetidas a ambientes contrastantes de irradiância aos 0,45 e 205 dias $(n=5) . A I=$ alta irradiância $(1900-2100$ $\left.\mu \mathrm{mol} \mathrm{m} \mathrm{m}^{-2} \mathrm{~s}^{-1}\right)$; $\mathrm{MI}=$ moderada irradiância $\left(800-1000 \mu \mathrm{mol} \mathrm{m}^{-2} \mathrm{~s}^{-1}\right)$ e BI = baixa irradiância $\left(20-300 \mu \mathrm{mol} \mathrm{m} \mathrm{m}^{-2} \mathrm{~s}^{-1}\right)$. A seta indica o início da diferença significativa entre os tratamentos $(\mathrm{P}<0,05)$. Os valores são médias \pm erro padrão.

FIGURE 2: Specific leaf area (AFE) in young plants of Bertholletia excelsa subjected to contrasting environments of irradiance to 0,45 and 205 days $(n=5)$. AI $=$ high irradiance $(1900-2100$ $\left.\mu \mathrm{mol} \mathrm{m} \mathrm{m}^{-2} \mathrm{~s}^{-1}\right) ; \mathrm{MI}=$ moderate irradiance $\left(800-1000 \mu \mathrm{mol} \mathrm{m}^{-2} \mathrm{~s}^{-1}\right)$ and $\mathrm{BI}=$ low irradiance $(20$ $\left.-300 \mu \mathrm{mol} \mathrm{m}^{-2} \mathrm{~s}^{-1}\right)$. The arrow indicates the start of significance difference between treatments $(\mathrm{P}<0.05)$. Values are means \pm standard error.

tratamentos AI e MI, enquanto a maior concentração de carotenoides em plantas sombreadas pode estar associada a um tipo específico de xantofila (luteína) ou caroteno ( $\alpha$-caroteno) que aprimoram a absorção da energia disponível (MATSUBARA et al., 2009). Em síntese, é evidente a plasticidade da estrutura do aparato fotossintético de plantas jovens de Bertholletia excelsa nas diferentes condições de irradiância, com aprimoramentos nas estratégias de captação da energia disponível, conforme observado em BI, e dissipação do excesso de energia em condições de moderada e alta irradiância como mecanismos preventivos de possíveis danos fotoinibitórios.

Os valores iniciais de fotossíntese líquida (A) foram em média $8 \mu \mathrm{mol} \mathrm{m}^{-2} \mathrm{~s}^{-1}$ e variaram entre 6,69 e $8,15 \mu \mathrm{mol} \mathrm{m}^{-2} \mathrm{~s}^{-1}$ (AI), 7,77 e 8,54 $\mu \mathrm{mol} \mathrm{m} \mathrm{m}^{-2} \mathrm{~s}^{-1}$ (MI) e 5,27 e 7,97 $\mu \mathrm{mol} \mathrm{m}^{-2} \mathrm{~s}^{-1}$ (BI) no decorrer do período experimental (Figura 4). A fotossíntese líquida foi influenciada pelo ambiente de luz $(\mathrm{P}<0,05)$, assim como houve efeito do período $(\mathrm{P}<0,05)$ e da interação entre os tratamentos e o período $(\mathrm{P}<0,05)$. As maiores diferenças entre os tratamentos foram observadas aos 50 dias de experimentação, quando os valores médios de A para o tratamento MI foram 1,5 vezes superiores aos observados em BI. Ao final de 120 dias, a fotossíntese líquida média em BI foi $20 \%$ e $30 \%$ vezes inferior aos tratamentos AI e MI, respectivamente. Os valores de fotossíntese líquida observados no presente estudo estão dentro da amplitude de valores reportados para a mesma espécie em plantio para a recuperação de área degradada (FERREIRA et al., 2009; 2012).

A plasticidade fisiológica da espécie arbórea tropical Swietenia macrophylla submetida a diferentes condições de irradiância é similar à observada para Bertholletia excelsa, uma vez que a 
espécie exibiu acentuado ganho de biomassa associado ao melhor desempenho fotossintético em condições de alta irradiância, bem como elevados teores de pigmentos cloroplastídicos quando exposta ao ambiente de baixa disponibilidade de luz (AZEVEDO; MARENCO, 2012; GONÇALVES et al., 2012). No entanto, Aniba rosaeodora, outra espécie arbórea tropical nativa da região amazônica, alcançou elevados níveis de assimilação de carbono e acúmulo de biomassa em condições intermediárias de

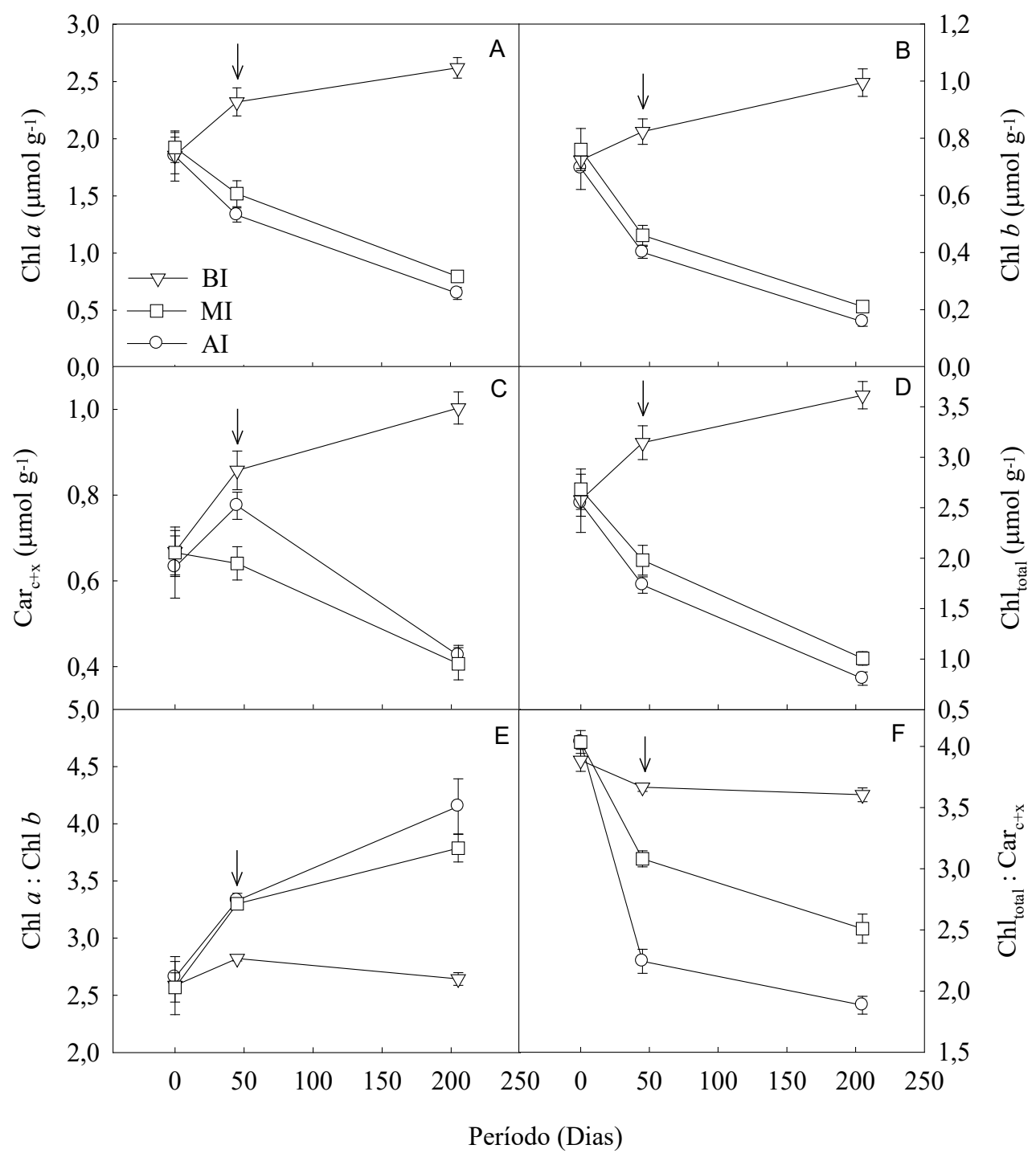

FIGURA 3: Teores foliares de clorofila $a$ (A), clorofila $b$ (B), carotenoides (C), clorofila total (D), razão clorofila $a / b(\mathrm{E})$ e razão clorofila total/carotenoides (F) em plantas jovens de Bertholletia excelsa submetidas a ambientes contrastantes de irradiância aos 0,45 e 205 dias $(n=5)$. AI $=$ alta irradiância $\left(1900-2100 \mu \mathrm{mol} \mathrm{m} \mathrm{m}^{-2} \mathrm{~s}^{-1}\right) ; \mathrm{MI}=$ moderada irradiância $(800-1000 \mu \mathrm{mol}$ $\left.\mathrm{m}^{-2} \mathrm{~s}^{-1}\right)$ e BI = baixa irradiância $\left(20-300 \mu \mathrm{mol} \mathrm{m}^{-2} \mathrm{~s}^{-1}\right)$. A seta indica o início da diferença significativa entre os tratamentos $(\mathrm{P}<0,05)$. Os valores são médias \pm erro padrão.

FIGURE 3: Leaf contents of chlorophyll $a(\mathrm{~A})$, chlorophyll $b$ (B), carotenoids (C), total chlorophyll (D) chlorophyll $a / b$ ratio (E) and total chlorophyll/carotenoids ratio (F) in young plants of Bertholletia excelsa subjected to contrasting environments of irradiance to 0, 45 e 205 days (n $=5) . \mathrm{AI}=$ high irradiance $\left(1900-2100 \mu \mathrm{mol} \mathrm{m}^{-2} \mathrm{~s}^{-1}\right) ; \mathrm{MI}=$ moderate irradiance $(800-1000$ $\left.\mu \mathrm{mol} \mathrm{m} \mathrm{s}^{-2} \mathrm{~s}^{-1}\right)$ and $\mathrm{BI}=$ low irradiance $\left(20-300 \mu \mathrm{mol} \mathrm{m} \mathrm{m}^{-2} \mathrm{~s}^{-1}\right)$. The arrow indicates the start of significance difference between treatments $(\mathrm{P}<0.05)$. Values are means \pm standard error. 


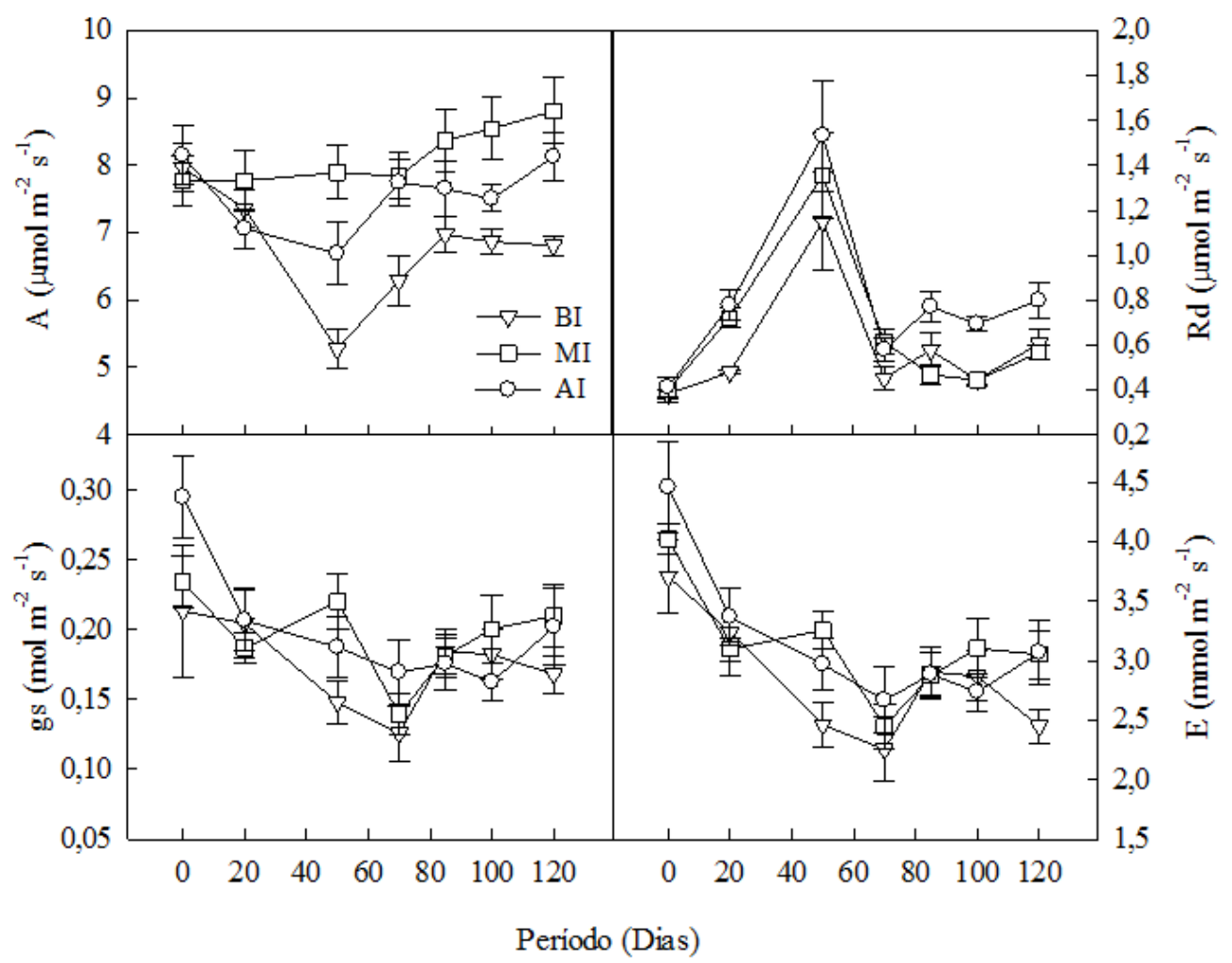

FIGURA 4: Fotossíntese líquida (A), respiração foliar no escuro (B), condutância estomática (C) e transpiração (D) em plantas jovens de Bertholletia excelsa submetidas a ambientes contrastantes de irradiância aos $0,20,50,70,85,100$ e 120 dias $(n=7) . A I=$ alta irradiância $(1900-2100$ $\left.\mu \mathrm{mol} \mathrm{m} \mathrm{m}^{-2} \mathrm{~s}^{-1}\right) ; \mathrm{MI}=$ moderada irradiância $\left(800-1000 \mu \mathrm{mol} \mathrm{m}^{-2} \mathrm{~s}^{-1}\right)$ e BI = baixa irradiância (20 - $\left.300 \mu \mathrm{mol} \mathrm{m}^{-2} \mathrm{~s}^{-1}\right)$. Os valores são médias \pm erro padrão.

FIGURE 4: Net photosynthesis (A), leaf dark respiration (B), stomatal conductance (C) and transpiration (D) in young plants of Bertholletia excelsa subjected to contrasting environments of irradiance to $0,20,50,85,100$ and 120 days $(\mathrm{n}=7)$. $\mathrm{AI}=$ high irradiance $\left(1900-2100 \mu \mathrm{mol} \mathrm{m} \mathrm{m}^{-2} \mathrm{~s}^{-1}\right)$; MI $=$ moderate irradiance $\left(800-1000 \mu \mathrm{mol} \mathrm{m}{ }^{-2} \mathrm{~s}^{-1}\right)$ e BI $=$ low irradiance $\left(20-300 \mu \mathrm{mol} \mathrm{m}^{-2} \mathrm{~s}^{-1}\right)$. Values are means \pm standard error.

irradiância, semelhante ao tratamento MI. Porém, quando exposta a níveis de irradiância mais elevados (1300-1800 $\mu \mathrm{mol} \mathrm{m} \mathrm{m}^{-2} \mathrm{~s}^{-1}$ ) observou-se fotoinibição, com redução da eficiência quântica e consequentemente da assimilação líquida de $\mathrm{CO}_{2}$ (GONÇALVES et al., 2005). Dentre outros fatores, a capacidade de utilizar e dissipar o excesso de energia de maneira eficiente é determinante para a assimilação líquida de $\mathrm{CO}_{2}$ e estabelecimento das espécies arbóreas em ambientes contrastantes de luz. Sendo assim, maiores taxas fotossintéticas em ambientes de alta irradiância são geralmente observadas em espécies com potencial de dissipar a energia excedente e garantir a manutenção e reparo do aparato fotossintético (AZEVEDO; MARENCO, 2012). Embora esse estudo não apresente resultados conclusivos acerca da eficiência de Bertholletia excelsa na dissipação da energia excedente, as informações obtidas em relação a $\mathrm{Chl}_{\text {total }} / \mathrm{C}_{\mathrm{c}+\mathrm{x}}$, desempenho fotossintético, crescimento e acúmulo de biomassa em condições de alta irradiância (1900$2100 \mu \mathrm{mol} \mathrm{m}^{-2} \mathrm{~s}^{-1}$ ) sugerem alta capacidade dessa espécie para regular e reparar o aparato fotossintético sob condições de irradiância saturante (FERREIRA et al., 2009). Ademais, a maior assimilação líquida de $\mathrm{CO}_{2}$ por unidade de área foliar em condições de irradiância saturante também tem sido atribuída à redução da área foliar específica. A maior quantidade de mesofilo, associada à menor AFE, possibilita o aumento da quantidade de cloroplastos e consequentemente da abundância da enzima carboxilativa (Rubisco) e dos carreadores de elétrons nas membranas do tilacoide na base de área foliar (KAMALUDDIN; GRACE, 1994; 
OGUCHI et al., 2005). Os menores valores de AFE, assim como a maior capacidade de lidar com a energia excedente em folhas de plantas submetidas aos ambientes de moderada e alta irradiância podem justificar a ocorrência e o pleno desenvolvimento de Bertholletia excelsa em áreas abertas e formações de clareiras na floresta (KAINER et al., 1998; OLIVEIRA, 2000; PEÑA-CLAROS et al., 2002; SOUZA et al., 2010; MOLL-ROCEK et al., 2014; SCOLES et al., 2014).

De modo geral, maiores valores de fotossíntese líquida em ambientes de alta irradiância são acompanhados por taxas de respiração foliar no escuro também superiores (WEERASINGHE et al., 2014). Dessa forma, a respiração foliar variou entre os tratamentos de irradiância $(\mathrm{P}<0,05)$ e ao longo dos 120 dias de monitoramento $(\mathrm{P}<0,05)$, apresentando valor máximo de $1,53 \mu \mathrm{mol} \mathrm{m}^{-2} \mathrm{~s}^{-1}$ aos 50 dias em AI e média mínima de $0,4 \mu \mathrm{mol} \mathrm{m}^{-2} \mathrm{~s}^{-1}$ para BI no início do período experimental. A condutância estomática variou com o decorrer do experimento $(\mathrm{F}=5,64, \mathrm{P}<0,05)$, com valor máximo de $0,3 \mathrm{~mol} \mathrm{~m}^{-2} \mathrm{~s}^{-1}(\mathrm{AI}-0$ dias) e mínimo de $0,13 \mathrm{~mol} \mathrm{~m}^{-2} \mathrm{~s}^{-1}$ aos 70 dias em BI, porém, sem efeito diferenciado dos tratamentos $(\mathrm{P}>0,05)$. Dessa forma, os valores contrastantes de fotossíntese entre os tratamentos parecem não ser causados por limitação estomática. Por sua vez, os valores de transpiração $(E)$ variaram entre os tratamentos $(\mathrm{P}<0,05)$ e no decorrer da exposição das plantas aos tratamentos $(\mathrm{P}<0,05)$. O maior valor de $\mathrm{E}\left(4,46 \mathrm{mmol} \mathrm{m}^{-2} \mathrm{~s}^{-1}\right)$ foi observado no tratamento AI no início do período experimental e a máxima redução dos valores foi observada aos 70 dias, com médias $64 \%, 68 \%$ e $64 \%$ inferiores às observadas na primeira análise ( 0 dias), para os tratamentos BI, MI e AI, respectivamente. Embora os valores de condutância estomática, intrínsecos à planta, não variaram entre os tratamentos, a menor umidade relativa do ar e, consequentemente maior demanda transpiratória, pode estar associada aos elevados valores de E nas plantas jovens de Bertholletia excelsa submetidas ao tratamento de alta irradiância.

\section{CONCLUSÕES}

Plantas jovens de Bertholletia excelsa exibem respostas ecofisiológicas que garantem sua plasticidade fenotípica em diferentes condições de irradiância. Sob níveis moderados a elevados de irradiância, a espécie reduz a área foliar específica, aumenta a assimilação de $\mathrm{CO}_{2}$, particiona maior quantidade de biomassa para as raízes e, assim, promove o crescimento e o acúmulo de biomassa. De outra forma, o estabelecimento e permanência das plântulas em médio prazo sob condições de baixa disponibilidade de irradiância é assegurado a baixas taxas de crescimento, porém, com balanço positivo de carbono, uma vez que a espécie aumenta a capacidade de interceptar e absorver os níveis limitantes de luz disponíveis.

\section{AGRADECIMENTOS}

Os autores dessa pesquisa agradecem à Universidade Federal do Amazonas (UFAM) pelo suporte logístico e infraestrutura dos experimentos, ao Laboratório de Fisiologia e Bioquímica Vegetal (LFBV/ INPA-MCTI) pela disponibilidade dos equipamentos e infraestrutura para a realização das análises e à Empresa Agropecuária Aruanã S. A. pela doação das mudas de Bertholletia excelsa no experimento. O Conselho Nacional de Desenvolvimento Científico e Tecnológico (CNPq) concedeu bolsas de mestrado e produtividade durante a pesquisa.

\section{REFERÊNCIAS}

AZEVEDO, G. F. C.; MARENCO, R. A. Growth and physiological changes in saplings of Minquartia guianensis and Swietenia macrophylla during acclimation to full sunlight. Photosynthetica, Praha, v. 50, n. 1, p. 86-94, mar. 2012.

BUGBEE, B. G. Growth, analises and yield components. In: SALISBURY, F. B. (Ed.). Units, symbols and terminology for plant physiology. Oxford: Oxford University Press, 1996. p. 115-119.

DAVANSO, V. M. et al. Photosynthesis, growth and development of Tabebuia avellanedae Lor. Ex Griseb. (Bignoniaceae) in flooded soil. Brazilian Archives of Biology and Technology, Curitiba, v. 45, n. 3, p. 375-384, sept. 2002.

D'OLIVEIRA, M. V.N. Artificial regeneration in gaps and skidding trails after mechanised forest exploitation 
in Acre, Brazil. Forest ecology and management, Amsterdam, v. 127, n. 1, p. 67-76, mar. 2000.

ESTEBAN, R. et al. Internal and external factors affecting photosynthetic pigment composition in plants: a meta-analytical approach. New Phytologist, Malden, v. 206, n. 1, p. 268-280. abr. 2014.

EVANS, J. R.; POOTER, H. Photosynthetic acclimation of plants to growth irradiance: the relative importance of specific leaf area and nitrogen partitioning in maximizing carbon gain. Plant, Cell $\&$ Environment, Oxford, v. 24, n. 8, p. 755-767, aug. 2001.

FERREIRA, M. J. et al. Photosynthetic parameters of young Brazil nut (Bertholletia excelsa HB) plants subjected to fertilization in a degraded area in Central Amazonia. Photosynthetica, Praha, v. 47, n. 4, p. 616-620, dec. 2009.

FERREIRA, M. J. et al. Crescimento e eficiência do uso da água de plantas jovens de Castanheira-daamazônia em área degradada e submetidas à adubação. Ciência Florestal, Santa Maria, v. 22, n. 2, p. 393-401, abr./jun. 2012.

GONÇALVES, J. F. C. et al. Growth, photosynthesis and stress indicators in young rosewood plants (Aniba rosaeodora Ducke) under different light intensities. Brazilian Journal of Plant Physiology, Londrina, v. 17, n. 3, p. 325-334, jul./set. 2005.

GONÇALVES, J. F. C. et al. Efeito do ambiente de luz no crescimento de plantas jovens de mogno (Swietenia macrophylla King). Scentia Florestalis, Piracicaba, v. 40, n. 95, p. 337-344, set. 2012.

HALLIK, L. et al. Photosynthetic acclimation to light in woody and herbaceous species: a comparison of leaf structure, pigment content and chlorophyll fluorescence characteristics measured in the field. Plant Biology, Freiburg, v. 14, n. 1, p. 88-99, jan. 2012.

HANSEN, U. et al. Variation of pigment composition and antioxidative systems along the canopy light gradient in a mixed beech/oak forest: a comparative study on deciduous tree species differing in shade tolerance. Trees, Berlin, v. 16, n. 4-5, p. 354-364, may 2002.

HAYASHIDA-OLIVER, Y. et al. Influencia de la disponibilidad de agua y luz en el crecimiento y la morfología de plantines de Swietenia macrophylla, Cedrela odorata y Bertholletia excelsa. Ecologia en Bolívia, La Paz, v. 35, p. 51-60, 2001.

HENDRY, G. A. F.; PRICE, A. H. Stress indicators: chlorophylls and carotenoids. In: HENDRY, G. A. F.; GRIME, J. P. (Eds). Methods in comparative plant ecology. London: Chapman and Hall, 1993.

KAINER, K. A. et al. Brazil nut seedling establishment and autecology in extractive reserves of Acre, Brazil. Ecological Applications, New York, v. 8, n. 2, p. 397-410, may 1998.

KAMALUDDIN, M.; GRACE, J. Growth and photosynthesis of tropical forest tree seedlings (Bischofia javanica Blume) as influenced by a change in light availability. Tree Physiology, Victoria, v. 13, n. 2, p. 189-201, set. 1993.

KENZO, T. et al. Height-related changes in leaf photosynthetic traits in diverse Bornean tropical rain forest trees. Oecologia, Berlin, v. 177, n. 1, p. 191-202, jan. 2015.

KITAJIMA, K.; HOGAN, K. P. Increases of chlorophyll a/b ratios during acclimation of tropical woody seedlings to nitrogen limitation and high light. Plant, cell \& environment, Oxford, v. 26, n. 6, p. 857-865, jun. 2003.

KRAUSE, G. H. et al. Photosynthesis, photoprotection, and growth of shade-tolerant tropical tree seedlings under full sunlight. Photosynthesis research, Tempe, v. 113, n. 1-3, p. 273-285, set. 2012.

LICHTENTHALER, H. K.; WELLBURN, A. Determination of total carotenoids and chlorophylls $a$ and $b$ of leaf extracts in different solvents. Biochemical Society Transactions, London, v. 11, p. 591-592, 1983. LICHTENTHALER, H. K. et al. Differences in pigment composition, photosynthetic rates and chlorophyll fluorescence images of sun and shade leaves of four tree species. Plant Physiology and Biochemistry, Bari, v. 45, n. 8, p. 577-588, aug. 2007.

LICHTENTHALER, H. K. et al. Chlorophyll fluorescence kinetics, photosynthetic activity, and pigment composition of blue-shade and half-shade leaves as compared to sun and shade leaves of different trees. Photosynthesis research, Tempe, v. 117, n. 1-3, p. 355-366, nov. 2013.

MATSUBARA, S. et al. Sun-shade patterns of leaf carotenoid composition in 86 species of neotropical forest plants. Functional Plant Biology, Melbourne, v. 36, p. 20-36, jan. 2009.

MOLL-ROCEK, J. et al. Brazil Nut (Bertholletia excelsa, Lecythidaceae) regeneration in logging gaps in the peruvian amazon. International Journal of Forestry Research, New York, v. 2014, p. 1-8, mar. 2014. 
MORI, S. A.; PRANCE, G. T. Taxonomy, ecology, and economic botany of the Brazil nut (Bertholletia excelsa humb. \& Bonpl.: Lecythidaceae). Advances in Economic Botany, New York, v. 8, p. 130-150, 1990.

MYERS, G. P. et al. The influence of canopy gap size on natural regeneration of Brazil nut (Bertholletia excelsa) in Bolivia. Forest Ecology and Management, Amsterdam, v. 127, n. 1-3, p. 119-128, mar. 2000. NIINEMETS, Ü. A review of light interception in plant stands from leaf to canopy in different plant functional types and in species with varying shade tolerance. Ecological Research, Tokyo, v. 25, n. 4, p. 693-714, jul. 2010.

OGUCHI, R. et al. Leaf anatomy as a constraint for photosynthetic acclimation: differential responses in leaf anatomy to increasing growth irradiance among three deciduous trees. Plant, Cell \& Environment, Oxford, v. 28, n. 7, p. 916-927, jul. 2005.

PEÑA-CLAROS, M. et al. Enrichment planting of Bertholletia excelsa in secondary forest in the Bolivian Amazon: effect of cutting line width on survival, growth and crown traits. Forest Ecology and Management, Amsterdam, v. 161, n. 1-3, p. 159-168, may 2002.

POORTER, H. et al. Biomass allocation to leaves, stems and roots: meta-analyses of interspecific variation and environmental control. New Phytologist, Malden, v. 193, n. 1, p. 30-50, jan. 2012.

POORTER, L. Light-dependent changes in biomass allocation and their importance for growth of rain forest tree species. Functional Ecology, London, v. 15, n. 1, p. 113-123, feb. 2001.

SANTOS JÚNIOR, U. M. et al. Growth, leaf nutrient concentration and photosynthetic nutrient use efficiency in tropical tree species planted in degraded areas in Central Amazonia. Forest Ecology and Management, Amsterdam, v. 226, n. 1-3, p. 299-309, may 2006.

SCHROTH, G. et al. Growth and nutrient accumulation of Brazil nut trees (Bertholletia excelsa) in agroforestry at different fertilizer levels. Journal of Forestry Research, Harbin, v. 26, n. 2, p. 347-353, jan. 2015.

SCOLES, R. et al. Crescimento e sobrevivência de castanheira (Bertholletia excelsa Bonpl., Lecythidaceae) plantada em diferentes condições de luminosidade após seis anos de plantio na região do rio Trombetas, Oriximiná, Pará. Boletim do Museu Paraense Emílio Goeldi. Ciências Naturais, Belém, v. 9, n. 2, p. 321-336, maio/ago. 2014.

SOUZA, C. R. et al. Comportamento de espécies florestais em plantios a pleno sol e em faixas de enriquecimento de capoeira na Amazônia. Acta Amazonica, Manaus, v. 40, n. 1, p. 127-134, mar. 2010.

STATSOFT. Statistica. 2010. Data Analysis Software System version 9.0.

SWAINE, M. D.; WHITMORE, T. C. On the definition of ecological species groups in tropical rain forests. Vegetatio, Dordrecht, v. 75, n. 1-2, p. 81-86, may 1988.

TONINI, H. et al. Relação da produção de sementes de castanha-do-Brasil com características morfométricas da copa e índices de competição. Pesquisa Agropecuária Brasileira, Brasília, v. 43, n. 11, p. 1509-1516, nov. 2008.

TRIPATHI, S. N.; RAGHUBANSHI, A. S. Seedling growth of five tropical dry forest tree species in relation to light and nitrogen gradients. Journal of Plant Ecology, Oxford, v. 7, n. 3, p. 250-263, jun. 2014.

WEERASINGHE, L. K. et al. Canopy position affects the relationships between leaf respiration and associated traits in a tropical rainforest in Far North Queensland. Tree physiology, Oxford, v. 34, n. 6, p. 564-584, apr. 2014.

WIENER, E. M. Ecological research and the management of Young successional forests: a case study on the reintroduction of native tree species on a Terra Firme site in northeastern Peru. Journal of Sustainable Forestry, New Haven, v. 29, n. 6-8, p. 571-590, oct. 2010.

ZUIDEMA, P. A. et al. Crecimiento de plantines de Bertholletia excelsa HBK em función de su tamaño y la disponibilidad de luz. Ecologia en Bolívia, La Paz, v. 33, p. 23-35, out. 1999. 